\title{
Oscillations of the Nernst coefficient in bismuth
}

\author{
Yu. V. Sharlai and G. P. Mikitik \\ B. Verkin Institute for Low Temperature Physics $\&$ Engineering, \\ Ukrainian Academy of Sciences, Kharkov 61103, Ukraine
}

\begin{abstract}
We calculate the magnetic-field dependence (oscillations) of the Nernst coefficient in bismuth at low temperatures for the case when the magnetic field is directed along the trigonal axis of the crystal. In the calculations we take into account the scattering of the electrons and holes in bismuth on impurities and the dependence of this scattering on the magnetic field. The results of these calculations are compared with the experimental data recently published.
\end{abstract}

PACS numbers: 72.15.Jf, 72.10.Di, 71.55.Ak

\section{INTRODUCTION}

In a recent paper, $\frac{1}{,}$ oscillations of the Nernst coefficient in bismuth were observed for magnetic fields directed along the trigonal and bisectrix axes of the crystal. These oscillations have the shape of rather sharp peaks which originate from the crossing of the Landau levels of charge carriers in bismuth with the Fermi level $E_{F}$ of this semimetal. Moreover, several unusual peaks of this coefficient were discovered for very high magnetic fields $H(14 \leq H \leq 33 \mathrm{~T})$ parallel to the trigonal axis $\stackrel{2}{2}$ At such magnetic fields almost all the Landau levels are empty, and one can hardly expect that the unusual peaks result from the above-mentioned crossing. So the authors of Ref. 2 suggested that the unusual peaks are caused by some collective effects in the electron system of bismuth. Interestingly, in the same interval of high magnetic fields several jumps of magnetization were observed, which were ascribed to field-induced instabilities of the ground state of interacting electrons in bismuth $\underline{3}$

Within a simple model of the electron energy spectrum of bismuth in magnetic fields, the positions of the usual peaks in the magnetic-field axis were explained,,,+ 5 by calculating the crossing of the appropriate Landau levels with $E_{F}$. It turned out that most of these peaks are due to the hole Fermi surface of bismuth, while some result from its electron Fermi surface. However, the unusual peaks observed in the Nernst signal at high magnetic fields cannot be explained in this way. Nevertheless, we showed 4 that in principle, the positions of some of these peaks can be reproduced if one assumes that a small deviation of the magnetic-field direction from the trigonal axis occurred in the experiments. In order to support or to disprove this possible explanation of the unusual peaks, we also suggested ${ }^{4}$ to investigate experimental investigation of the dependence of the Nernst-coefficient peaks on the tilt angle of the magnetic field. These experiments were described in Ref. 6, and it was found that the angular dependences of the usual peaks are in agreement with the theory, $\stackrel{4,5}{ }$ while the dependences of the unusual peaks are essentially different, and hence their origin cannot be explained by a small deviation of the magnetic-field direction from the trigonal axis. In addition, the results of the calculations in Ref. 4 show that the usual electron peaks observed in the experiment correspond to only some of the crossings of the electron Landau levels with $E_{F}$, while other crossings do not manifest themselves.

In order to continue analysis of the experimental findings,,$\frac{1,2,6}{6}$ in this paper we calculate not only the positions of the peaks in the Nernst coefficient but also their shape, i.e. the dependence of this coefficient on the magnetic field, in the case when the field is along the trigonal axis. We carry out the calculations within the same model of the spectrum of bismuth as in Ref. 4. This model is briefly described in Sec. II. In Sec. III we present formulas for the Nernst coefficient, taking into account the effect of impurities on the density of states of charge carriers in bismuth, and in Sec. IV we compare the results of our calculations with the experimental data.

\section{ENERGY SPECTRUM OF BISMUTH}

The Fermi surface of bismuth consists of one hole ellipsoid located at the point $\mathrm{T}$ of its Brillouin zone and three electron ellipsoids centered at the points $\mathrm{L}$ and elongated along the bisectrix directions $\stackrel{7}{7}$ As in Ref. 4, we shall describe the electron spectrum of bismuth in the magnetic field $\mathbf{H}$ by the model of Smith, Baraff and Rowell. $\stackrel{8}{-}$ According to this model, the $n$th Landau level $\varepsilon_{n}$ for an electron with the quasimomentum $p_{H}$ along $\mathbf{H}$ can be found from the equation:

$$
E\left(1+\frac{E}{E_{g}}\right)=\left(n+\frac{1}{2}\right) \hbar \omega_{c}+\frac{p_{H}^{2}}{2 m_{H}} \pm \frac{1}{2} g \beta_{0} H,
$$

where the signs \pm correspond to the electron spins that are antiparallel and parallel to $\mathbf{H}$, respectively; the energy $E$ is measured from the edge of the conduction band; $\omega_{c}$ is the cyclotron frequency

$$
\omega_{c}=\frac{|e| H}{m_{c} c},
$$

$E_{g}$ is the gap between the conduction and valence bands at the point $\mathrm{L} ; g$ is the effective electron $\mathrm{g}$ factor at this point; $\beta_{0}$ is the Bohr magneton; $c$ is the speed of light; $e$ is the electron charge, and the longitudinal and the 
TABLE I. Parameters of the Smith-Baraff-Rowell spectrum ${ }^{8}$

\begin{tabular}{lcccc}
\hline \hline Electrons & $m_{11}$ & $m_{22}$ & $m_{33}$ & $m_{23}$ \\
\hline Orbital mass & 0.00113 & 0.26 & 0.00443 & -0.0195 \\
Spin mass & 0.00101 & 2.12 & 0.0109 & -0.13 \\
\hline Holes & $M_{1}=M_{2}$ & $M_{3}$ \\
\hline Orbital mass & 0.07 & 0.69 \\
Spin mass & 0.033 & 200 \\
\hline$E_{g}=15.3 \mathrm{meV}$ & \multicolumn{3}{c}{$E_{0}=38.5 \mathrm{meV}$} \\
\hline \hline
\end{tabular}

cyclotron masses, $m_{H}$ and $m_{c}$, are given by

$$
\begin{aligned}
m_{H} & =\mathbf{h} \cdot \mathbf{m}^{e} \cdot \mathbf{h}, \\
m_{c} & =\left[\operatorname{det} \mathbf{m}^{e} / m_{H}\right]^{1 / 2} .
\end{aligned}
$$

Here $\mathbf{h}$ is the unit vector in the direction of the magnetic field $\mathbf{H}$. The effective mass tensor $\mathbf{m}^{e}$ has the form:

$$
\mathbf{m}^{e}=\left(\begin{array}{ccc}
m_{11} & 0 & 0 \\
0 & m_{22} & m_{23} \\
0 & m_{23} & m_{33}
\end{array}\right)
$$

where the axes 1 and 3 coincide with the binary and the trigonal axes, respectively, while the axis 2 is along the bisectrix direction. The effective $g$ factor,

$$
g^{2}=4 m^{2} \frac{\mathbf{h} \cdot \mathbf{m}_{s}^{e} \cdot \mathbf{h}}{\operatorname{det} \mathbf{m}_{s}^{e}}
$$

is defined in terms of a spin-mass tensor $\mathbf{m}_{s}^{e}$ that has the form similar to Eq. (41). Within the model of Smith, Baraff, and Rowell $\underline{\underline{8}}$ the elements of $\mathbf{m}_{s}^{e}$ differ from the elements of $\mathbf{m}^{e}$.

Since the spectrum of the holes at the point $\mathrm{T}$ is parabolic, their Landau levels can be easily found ${ }^{7}$

$$
E_{0}-E=\left(n+\frac{1}{2}\right) \hbar \omega_{c}+\frac{p_{H}^{2}}{2 m_{H}} \pm \frac{1}{2} g \beta_{0} H,
$$

where $E_{0}$ is the edge of the hole band at this point of the Brilloun zone. The cyclotron frequency $\omega_{c}$, the masses $m_{c}$ and $m_{H}$, and the $g$ factor are defined by the same formulas (2), (3), (5) as for the electrons, but now the tensor of the effective masses for the holes $\mathbf{m}^{h}$ has the form:

$$
\mathbf{m}^{h}=\left(\begin{array}{ccc}
M_{1} & 0 & 0 \\
0 & M_{2} & 0 \\
0 & 0 & M_{3}
\end{array}\right),
$$

and a similar expression is valid for the spin-mass tensor, $\mathbf{m}_{s}^{h}$. All the parameters in Eqs. (1) - (7) are known for bismuth; $;, 9$ see Table I.

The above formulas lead to the following expression for the contribution of the i-th electron ellipsoid to the density of electrons in bismuth,,

$$
N_{i}^{e}\left(E_{F}, H\right)=\frac{\left(2 m_{H}\right)^{1 / 2} e H}{2 \pi^{2} \hbar^{2} c} \sum_{n, \pm} \sqrt{E_{F}^{*}-E_{n, \pm}},
$$

where

$$
E_{F}^{*}=E_{F}\left(1+E_{F} / E_{g}\right)
$$

$E_{F}$ is the Fermi energy, and we have used the notation

$$
E_{n, \pm}=(n+1 / 2) \hbar \omega_{c} \pm(1 / 2) \beta_{0} g H .
$$

The density of the holes is given by ${ }^{8}$

$$
N^{h}\left(E_{F}, H\right)=\frac{\left(2 m_{H}\right)^{1 / 2} e H}{2 \pi^{2} \hbar^{2} c} \sum_{n, \pm} \sqrt{E_{0}-E_{F}-E_{n, \pm}} .
$$

The position of the Fermi energy $E_{F}(H)$ is determined by the condition of charge neutrality:

$$
Z \equiv \sum_{i=1}^{3} N_{i}^{e}\left(E_{F}, H\right)-N^{h}\left(E_{F}, H\right)=0 .
$$

Below we shall apply the formulas of this section to the case when the magnetic field is directed along the trigonal axis of the crystal, i.e., when $\mathbf{h}=(0,0,1)$.

\section{NERNST COEFFICIENT}

Let the $z$ axis of the coordinate system be directed along the trigonal axis of the crystal, while the $x$ and $y$ axes lie in the basal plane of bismuth. In the presence of a weak electric field $\mathbf{E}$ and of a small temperature gradient $\nabla T$, the charge-current density $\mathbf{j}$ is determined by

$$
j_{i}=\sigma_{i k} E_{k}^{*}-\alpha_{i k} \nabla_{k} T
$$

where the subscripts $i, k$ stand for the spatial coordinates $x, y, z ; \mathbf{E}^{*}=\mathbf{E}+\frac{1}{e} \nabla \mu ; \mu$ is the local chemical potential in the crystal; $\sigma_{i k}$ and $\alpha_{i k}$ are the conductivity and Peltier (thermoelectric) tensors, respectively. In the absence of an external magnetic field the tensors $\sigma_{i k}$ and $\alpha_{i k}$ are diagonal, and one has

$$
\sigma_{x x}=\sigma_{y y}, \quad \alpha_{x x}=\alpha_{y y} .
$$

If the external magnetic field $\mathbf{H}$ is applied along the $z$ axis, the off-diagonal terms $\sigma_{x y}, \sigma_{y x}$ and $\alpha_{x y}, \alpha_{y x}$ become different from zero, and they satisfy the relationships:

$$
\sigma_{x y}=-\sigma_{y x}, \quad \alpha_{x y}=-\alpha_{y x} .
$$

In this situation the temperature gradient $\nabla_{x} T$ will generate a transverse electric field $E_{y}$. The Nernst coefficient $\nu$ is defined by the relation

$$
\nu H=\left|\frac{E_{y}}{\nabla_{x} T}\right| \mathbf{j = 0},
$$

and it can be found from the formula

$$
S_{x y} \equiv \nu H=\left|\frac{\sigma_{x x} \alpha_{x y}-\sigma_{x y} \alpha_{x x}}{\sigma_{x x}^{2}+\sigma_{x y}^{2}}\right|,
$$


which immediately follows from Eqs. (11)-(14).

Since the oscillations of the Nernst coefficient can be observed only at sufficiently high magnetic fields, we assume below that the condition

$$
\omega_{c} \tau \gg 1
$$

is fulfilled for both the electrons and the holes in bismuth where $\tau$ is the scattering time of an electron or a hole on impurities. In clean crystals of bismuth condition (16) can be satisfied even for moderate magnetic fields. For example, using the parameters presented in Table I and the experimental data of Ref. 10, we estimate that at $H=1 \mathrm{kOe}$ directed along the trigonal axis the values of $\omega_{c} \tau$ for the electrons and holes are of the order of 10 for the crystal used by K. Behnia et al $\underline{\underline{10}}$ (here we have assumed that the scattering lengths for the holes and the electrons are approximately the same).

Under condition (16), the diagonal component $\alpha_{x x}$ of the Peltier tensor is small as compared with $\alpha_{x y}: 11$

$$
\frac{\alpha_{x x}}{\alpha_{x y}} \sim \frac{1}{\omega_{c} \tau} \ll 1
$$

Since bismuth has equal numbers of electrons and holes, the ratio $\sigma_{x y} / \sigma_{x x}$ in this semimetal is also suppressed as compared to the case of a metal with a single type of charge carrier ${ }^{12}$ (e.g., in Ref. $13 \sigma_{x x}$ was approximately ten times larger than $\sigma_{x y}$ ). Then, we obtain from Eq. (15) that

$$
\nu H \approx \frac{\sigma_{x x} \alpha_{x y}}{\sigma_{x x}^{2}+\sigma_{x y}^{2}}=\rho_{x x} \alpha_{x y},
$$

where $\rho_{i k}=\sigma_{i k}^{-1}$ is the resistivity tensor. As the experimental data ${ }^{14}$ show, $\rho_{x x}$ is a smooth and almost monotonic function of the magnetic field in that region of the $T-H$ plane in which the oscillations of the Nernst coefficient were observed. $\stackrel{1,2}{=}$ Hence, the peaks in the Nernst coefficient mainly result from the peaks in $\alpha_{x y}$, and below we shall analyze only this $\alpha_{x y}$, taking $\rho_{x x}(H)$ from the experiment $\underline{\underline{14}}$

The off-diagonal term of the Peltier tensor, $\alpha_{x y}$, consists of the purely electronic and the phonon-drag parts,

$$
\alpha_{x y}=\alpha_{x y}^{(e)}+\alpha_{x y}^{(p h)} .
$$

The purely electronic part $\alpha_{x y}^{(e)}$ is caused by a nonequilibrium distribution of the electrons and holes over the crystal when $\nabla_{x} T \neq 0$. In strong magnetic fields, $\omega_{c} \tau \gg 1$, $\alpha_{x y}^{(e)}$ is dissipationless, i.e. it does not depend on the relaxation mechanisms of this distribution: 15,16

$\alpha_{x y}^{(e)}=\frac{c S}{H}=\frac{c}{H} \int_{-\infty}^{+\infty} d E \frac{\partial Z}{\partial E}\left\{f_{F} \ln f_{F}+\left(1-f_{F}\right) \ln \left(1-f_{F}\right)\right\}$,

where $S$ is the entropy of the charge carriers per a unit volume; $f_{F}$ is the Fermi function with the chemical potential $\mu$, and $\partial Z / \partial E$ is the density of states of the charge carriers in bismuth,

$$
\frac{\partial Z(E, H)}{\partial E}=\sum_{i=1}^{3} \frac{\partial N_{i}^{e}(E, H)}{\partial E}+\left|\frac{\partial N^{h}(E, H)}{\partial E}\right| .
$$

According to formulas (19), (6), (8), and (9), the derivative $\partial Z / \partial E$ diverges when the energy $E$ crosses the Landau levels. The integral over $E$ with the hat-like function $\left\{f_{F} \ln f_{F}+\left(1-f_{F}\right) \ln \left(1-f_{F}\right)\right\}$ of characteristic width $T$ smooths the singularities of $\partial Z / \partial E$, but at low temperatures, $\hbar \omega_{c} \gg T$, the quantity $\alpha_{x y}^{(e)}$ shows sharp peaks when the Fermi energy approaches a Landau level. These simple considerations qualitatively explain the origin of the peaks in the Nernst coefficient of bismuth.

The phonon-drag part $\alpha_{x y}^{(p h)}$ originates from the charge carriers that are dragged by a phonon flow in the crystal due to the electron-phonon interaction. In the strong magnetic fields this part is described by a sufficiently complicated formula which schematically has the form: 17,18

$$
\alpha_{x y}^{(p h)} \propto \frac{c}{H}\left\langle\frac{\nu_{p e}}{\nu_{p e}+\nu_{0}}\right\rangle,
$$

where $\nu_{p e}$ is the probability of phonon scattering by the charge carriers; $\nu_{0}$ is the probability of phonon scattering by other phonons and by the boundaries of the sample; $\langle\ldots\rangle$ means some averaging over the wave vectors of the phonons existing at the temperature $T$. It is essential here that the probability $\nu_{p e}$ is proportional to the density of states $\partial Z / \partial E$ and oscillates with changing $H$. At very low temperatures the phonon scattering by the boundaries of the sample prevails over other phonon scattering processes,$\frac{10}{-}$ i.e., one has $\nu_{0} \gg \nu_{p e}$. In this case the expression for $\alpha_{x y}^{(p h)}$ is proportional to $\partial Z / \partial E$ and is similar to Eq. (20). In order to estimate the magnetic-field dependence of the phonon-drag part in this situation, we shall use the simplest approximation

$$
\alpha_{x y}^{(p h)}(H, T) \approx r(T) \alpha_{x y}^{(e)}(H, T),
$$

where the factor $r(T)$ can be found as the ratio of $\alpha_{x y}^{(p h)}$ and $\alpha_{x y}^{(e)}$ at sufficiently low magnetic fields when the oscillations of $\alpha_{x y}$ become small. With increasing $T$ the probability $\nu_{p e}$ may become of the order of $\nu_{0}$ or even exceed it. According to Ref. 10, this seems to occur in bismuth at $T \sim 3-4 \mathrm{~K}$. Then, $\nu_{p e} /\left(\nu_{p e}+\nu_{0}\right) \sim 1$, and the oscillations of $\partial Z / \partial E$ with changing $H$ scarcely reveal themselves in $\alpha_{x y}^{(p h)}$.

\section{A. Effect of impurities on the electron and hole densities of states in bismuth}

Although in the limit of strong magnetic fields, $\omega_{c} \tau \gg$ 1 , formula (20) does not depend on $\tau$ explicitly, the scattering of the charge carriers on impurities renormalizes the density of states of the electrons and holes in bismuth, 
causing a smearing of the peaks in $\alpha_{x y}$. We calculate the renormalized $\partial Z / \partial E$ for bismuth using the approach of Ref. 19 .

Within this approach Green functions $G_{l}$ of charge carriers in the magnetic field $H$ are considered that are averaged over the positions of impurities in the crystal. The index $l$ means the set of quantum numbers $n, p_{z}, p_{y}$, $s_{z}= \pm 1$ that characterize eigenstates of a charged particle in a magnetic field in the absence of impurities. The potential of an impurity, $U(\mathbf{r})$, is assumed to be of the form $U(\mathbf{r})=f \delta(\mathbf{r})$ where $\delta(\mathbf{r})$ is the delta function, and the constant factor $f$ determines the scattering amplitude of a charged particle on an impurity at $\varepsilon=0$ and $H=0$. The density of states of the charge carriers per unit interval of their energy $\varepsilon, \partial N(\varepsilon) / \partial \varepsilon$, is expressed in terms of the Green functions as

$$
\frac{\partial N}{\partial \varepsilon}=\frac{1}{\pi} \operatorname{Im} G(\varepsilon),
$$

where $G(\varepsilon)=\sum_{l} G_{l}(\varepsilon)$. The function $G(\varepsilon)$ can be found from the self-consistent equation ${ }^{19}$

$$
G=\sum_{l}\left(\varepsilon_{l}-\varepsilon-c_{i} f^{2} G\right)^{-1},
$$

where $\varepsilon_{l}=\varepsilon\left(n, p_{z}, s_{z}\right)$ is the spectrum of a charge carrier in the magnetic field in the absence of impurities, and $c_{i}$ is the impurity concentration. The quantity $f$ can be expressed in terms of the scattering time $\tau$ of the electrons (the holes) at $H=0$ when the energy $\varepsilon$ of these particles is equal to the chemical potential $\mu_{0}$ of bismuth in zero magnetic field:

$$
\left.c_{i} f^{2} G\right|_{H \rightarrow 0, \varepsilon \rightarrow \mu_{0}}=i \frac{\pi \hbar}{2 \tau} .
$$

Below we calculate $G$ under the simplification that the scattering of the holes and of the electrons of the three different ellipsoids of bismuth does not mix these particles, and so one has electron $\tau_{e}$ and hole $\tau_{h}$ scattering times.

The integration over $p_{z}$ and $p_{y}$ in Eq. (25) can be done explicitly for the electrons and holes that are described by the spectra (11) and (6), respectively. Then, in the case of the holes we obtain the following equation in $G$ :

$$
G-G_{0}=i \frac{m_{z}^{1 / 2} e H}{2^{5 / 2} \pi \hbar^{2} c} \sum_{n, \pm}\left\{g_{n, \pm}\left(c_{i} f^{2} G\right)-g_{n, \pm}(0)\right\}
$$

where

$$
g_{n, \pm}(x)=\frac{1}{\sqrt{E_{0}-\varepsilon+x-E_{n, \pm}}}
$$

and $G_{0}(\varepsilon)$ is the function $G(\varepsilon)$ in the absence of impurities. The function $G_{0}$ is imaginary and is given by

$$
\operatorname{Im} G_{0}=\pi \frac{\partial N_{0}^{h}(\varepsilon, H)}{\partial \varepsilon}
$$

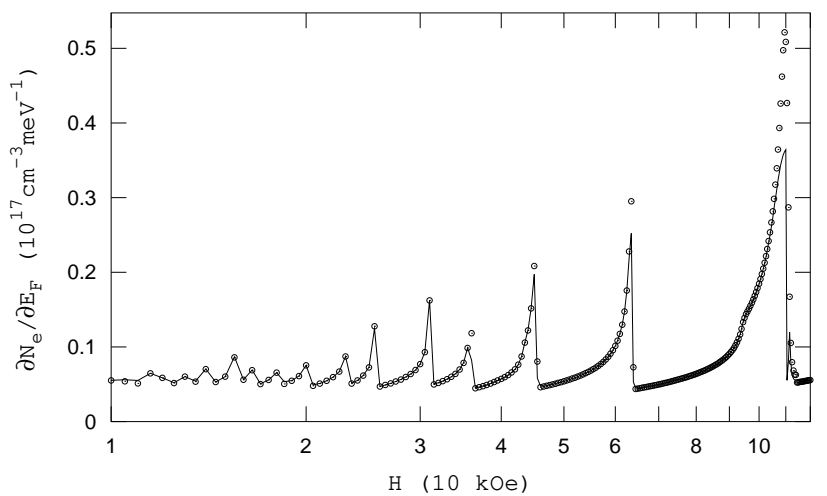

FIG. 1. The density of states for the electrons in bismuth. The solid line has been calculated with Eqs. (24)- (31) and $\hbar / \tau_{e}=0.001 \mathrm{meV}$. The points show the analytical result obtained with the substitution (32) at $\hbar / \tau_{e}^{\prime}=0.1 \mathrm{meV}$.

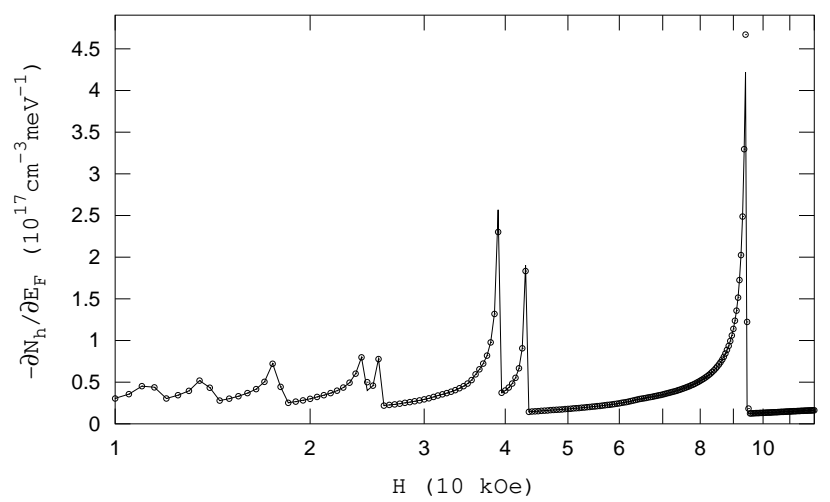

FIG. 2. The density of states for the holes in bismuth. The solid line has been calculated with Eqs. (24) - (31) and $\hbar / \tau_{h}=$ $0.5 \cdot 10^{-3} \mathrm{meV}$. The points show the analytical result obtained with the substitution (32) at $\hbar / \tau_{h}^{\prime}=0.007 \mathrm{meV}$.

where $\partial N_{0}^{h} / \partial \varepsilon$ is the density of states of the holes in the absence of impurities, i.e, $\partial N_{0}^{h} / \partial \varepsilon$ directly follows from Eq. (9). The functions $G$ for all the electron groups are identical, and the equation in $G$ has the same form as Eq. (27) but with

$$
g_{n, \pm}(x)=\frac{E_{g}+2(\varepsilon+x)}{E_{g}^{1 / 2}\left[(\varepsilon+x)^{2}+E_{g}\left(\varepsilon+x-E_{n, \pm}\right)\right]^{1 / 2}},
$$

and

$$
\operatorname{Im} G_{0}=\pi \frac{\partial N_{0}^{e}(\varepsilon, H)}{\partial \varepsilon},
$$

where $\partial N_{0}^{e} / \partial \varepsilon$ is the density of states of one of the electron ellipsoids in absence of the impurities. The renormalized densities of states of the electrons and of the holes in bismuth calculated with equations (24) - (31) are shown in Figs. 1 and 2.

Interestingly, the renormalized densities of states can be approximately obtained by the following simple sub- 
stitution in formulas (8), (9):

$$
u \rightarrow \frac{\sqrt{u^{2}+\Gamma^{2}}+u}{2},
$$

and by the subsequent differentiation of these formulas over $E_{F}$. Here $\Gamma=\hbar / \tau^{\prime}, u=E_{0}-E_{F}-E_{n, \pm}$ for the holes and $u=E_{F}^{*}-E_{n, \pm}$ for the electrons. It turns out that at an appropriate choice of the effective scattering times $\tau^{\prime}$, the densities of state thus obtained very well describe the densities of states calculated with Eqs. (24)- (31), see Figs. 1 and 2. The effective scattering time $\tau^{\prime}$ weakly depends on the number $n$ of the Landau level nearest to $E_{F}$, and so one and the same value of $\tau^{\prime}$ can describe the oscillations of the density of states in a rather wide interval of the magnetic fields, Figs. 1 and 2. Note that $\tau^{\prime}$ found for strong magnetic fields is essentially larger than $\tau$ defined in the limit $H \rightarrow 0$.

The substitution (32) can be understood from the following considerations: Let us average the density of states of charge carriers in the absence of impurities, $\partial N_{0}(E) / \partial E$, with a hat-like function characterized by some half-width $\Gamma=\hbar / \tau^{\prime}$ :

$$
\frac{\partial N(\varepsilon)}{\partial \varepsilon}=\frac{1}{\pi} \int_{-\infty}^{\infty} d E \frac{\left(\hbar / \tau^{\prime}\right)}{(E-\varepsilon)^{2}+\left(\hbar / \tau^{\prime}\right)^{2}} \frac{\partial N_{0}(E)}{\partial E} .
$$

In the case of the holes in bismuth (and for any quadratic spectrum) this integral is calculated analytically, and the density of states obtained coincides with $\partial N\left(E_{F}\right) / \partial E_{F}$ calculated with the substitution (32).

Using the substitution, one can easily understand the result that follows from the calculations with Eqs. (24)(31): In the presence of impurities, the positions of the peaks in $\partial N\left(E_{F}\right) / \partial E_{F}$ generally do not coincide with the magnetic fields at which the Landau levels calculated at zero impurity concentration, $c_{i}=0$, cross the Fermi energy. Indeed, for the holes in bismuth, we find from formula (9) with substitution (32) that

$$
\left|\frac{\partial N^{h}}{\partial E_{F}}\right| \approx \frac{\left(2 m_{z}\right)^{1 / 2} e H}{4 \sqrt{2} \pi^{2} \hbar^{2} c} \sum_{n, \pm} \frac{\sqrt{\sqrt{u_{n, \pm}^{2}+\Gamma^{2}}+u_{n, \pm}}}{\sqrt{u_{n, \pm}^{2}+\Gamma^{2}}}
$$

where $u_{n, \pm}=E_{0}-E_{F}-E_{n, \pm}$. Now each term of this sum reaches its maximum at $u_{n, \pm}=\Gamma / \sqrt{3}$. On the other hand, in the absence of impurities the singularities in $\partial N_{0}\left(E_{F}\right) / \partial E_{F}$ occur at $u_{n, \pm}=0$. In other words, if one takes into account the impurities, the positions of the peaks in the density of states shift.

\section{RESULTS}

In Figs. 36 6] we show the magnetic-field dependences of the quantity $S_{x y}=\nu H$ calculated with Eqs. (18) - (31) at different temperatures $T$, using the data on the resistivity $\rho_{x x}(H)$ measured in Ref. 14. For comparison, in

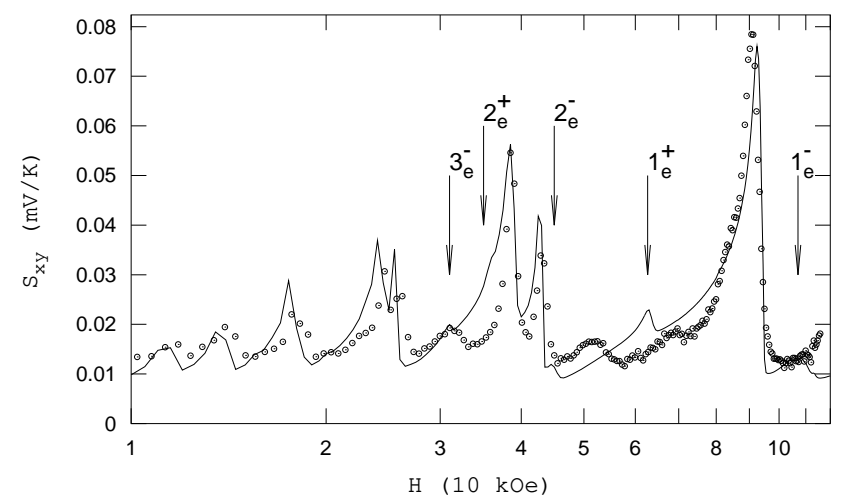

FIG. 3. The Nernst signal $S_{x y}=\nu H$ as a function of the magnetic field, $H$, at $T=0.18 \mathrm{~K}$. The points show the experimental data, $\frac{6}{-}$ and the solid line is the result of the calculation with Eqs. (18) - (31). Here $r(0.18)=0.85, \hbar / \tau_{h}=0.005 \mathrm{meV}$, and $\hbar / \tau_{e}=0.005 \mathrm{meV}$. The arrows indicate positions of the electron peaks visible in the solid line.

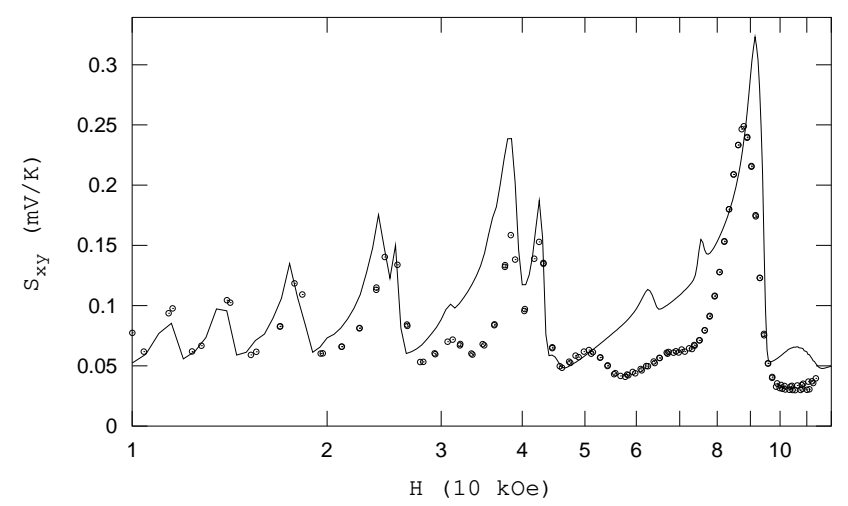

FIG. 4. As Fig. 3 but here $T=0.49 \mathrm{~K}$ and $r(0.49)=2.5$.

these figures we also present the appropriate experimental data ${ }^{6}$ for $S_{x y}(H)$. In the calculation of the curves $S_{x y}(H)$ we take into account the $H$-dependence of the chemical potential $\mu$ which is determined by Eq. (10). Since the renormalization of the electron and hole densities of states by impurities influences this dependence, we have calculated $N^{h}$ and $N_{i}^{e}$ in equation (10) using formulas (8), (9) and substitution (32). At temperatures $T=0.18 \mathrm{~K}, 0.49 \mathrm{~K}$ and $1.04 \mathrm{~K}$ the factor $r$ in Eq. (23) has been chosen in such a way that the calculated and the experimental curves coincide at a point near the lowest magnetic field $H \approx 10 \mathrm{kOe}$, i.e. at the point where the oscillations of $S_{x y}(H)$ are small. At $T=4.3 \mathrm{~K}$ (Fig. 6), in accordance with the considerations of Sec. III, we take $S_{x y}^{(p h)}(H)$ as a constant. The value of this constant, $S_{x y}^{(p h)}=0.21 \mathrm{mV} / \mathrm{K}$, has been found from the condition that the calculated $S_{x y}$ at $H=10 \mathrm{kOe}$ fits the appropriate experimental value.

An inspection of Figs. 36 shows that the theoretical curves sufficiently well describe the experimental data especially in the low-field part of the figures where the 


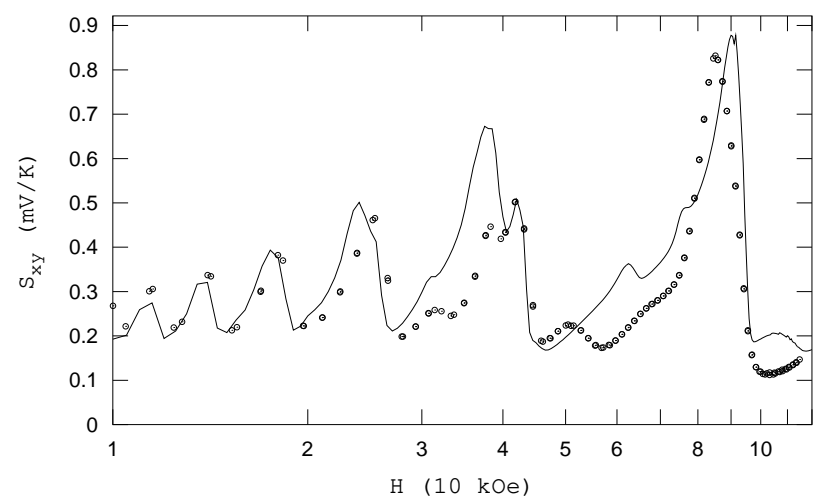

FIG. 5. As Fig. 3 but here $T=1.04 \mathrm{~K}$ and $r(1.04)=4.5$.

electron peaks essentially do not manifest themselves. Note also that the values $\tau_{h}=\tau_{e} \approx 1.2 \cdot 10^{-10} \mathrm{sec}$ used in the construction of these figures are of the same order of magnitude as those estimated in Ref. 20 from the data of transport measurements $\left(\tau_{h} \approx 8.5 \cdot 10^{-10} \mathrm{sec}\right.$ and $\tau_{e} \approx 4.4 \cdot 10^{-10} \mathrm{sec}$ ). Interestingly, although $\tau_{e}$ is equal to $\tau_{h}$ here, we find a relatively small amplitude for the electron peaks since the hole density of states is essentially larger than the electron contribution to $\partial Z\left(E_{F}\right) / \partial E_{F}$. That is why only a few electron peaks are seen in the experiment,,$\frac{6}{\underline{a}}$ and the value of $\tau_{e}$ has a weak effect on the calculated $S_{x y}$. The visible difference in the positions of the electron peaks in the theoretical and experimental curves seems to be caused by an insufficient accuracy of

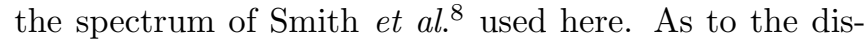
crepancy between the hole peaks in the theoretical and experimental curves in the high-field part of the figures, it could be due to the two reasons. First, our simplest approximation for the phonon-drag part $\alpha_{x y}^{(p h)}$, Eq. (23), and for $S_{x y}^{(p h)}(T=4.3 \mathrm{~K})$ [i.e., $S_{x y}^{(p h)}(T=4.3 \mathrm{~K})=0.21$ $\mathrm{mV} / \mathrm{K}]$ is probably impaired as magnetic field $H$ deviates farther and farther away from the point $10 \mathrm{kOe}$ at which the factors $r$ and the value of $S_{x y}^{(p h)}(T=4.3 \mathrm{~K})$ were found. Second, equation (25) obtained in Ref. 19, strictly speaking, is valid in the semiclassical limit (when the Landau-level number $n \gg 1$ ), while we use this equation in the whole interval of the magnetic fields. In other words, in the ultraquantum limit $(n \sim 1)$ a special analysis of $S_{x y}$ is required.

In the inset of Fig. [6 we compare the values of $S_{x y}$ calculated at $H=1 \mathrm{kOe}$ for $T=0.18 \mathrm{~K}, 0.49 \mathrm{~K}, 1.04$ $\mathrm{K}$, and $4.3 \mathrm{~K}$ with the experimental temperature dependence of $S_{x y}$ measured in Ref. 10 at the same magnetic field. These two functions $S_{x y}(T)$ are seen to agree reasonably well if it is remembered that they correspond to different crystals of bismuth [the phonon-drag part $S_{x y}^{(p h)}$ depends on the dimensions of the sample at low temperatures when the phonon mean-free path $l \propto\left(\nu_{p e}+\nu_{0}\right)^{-1}$ is set by the sample size].

The values of the factor $r$ obtained (see the captions to Figs. 315) demonstrate that the relative contribution

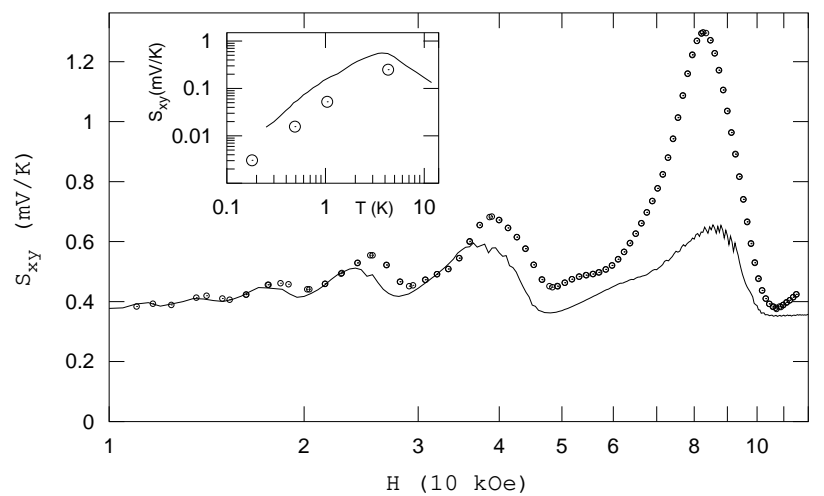

FIG. 6. As Fig. 3 but here $T=4.3 \mathrm{~K}$ and instead of formula (23) we use $S_{x y}^{(p h)}(H)=0.21 \mathrm{mV} / \mathrm{K}$. The inset shows the function $S_{x y}(T)$ at $H=1 \mathrm{kOe}$ (the solid line represents the experimental data, ${ }^{10}$ while the points are the values calculated here).

of the phonon-drag part $S_{x y}^{(p h)}$ to $S_{x y}$ decreases with decreasing $T$. Our estimates show that at $T \leq 0.05 \mathrm{~K}$ the phonon-drag part will practically die out, and one will be able to neglect $S_{x y}^{(p h)}$ completely in the calculations and hence to compare directly the electronic part $S_{x y}^{(e)}(H)$ with the experimental data. Therefore, the measurements of the Nernst signal at such low temperatures could provide the possibility to compare the theory and the experiment more accurately.

\section{CONCLUSIONS}

We calculate the purely electronic part $S_{x y}^{(e)}(H)$ of the Nernst signal in bismuth at low temperatures for the case when the magnetic field is directed along the trigonal axis of the crystal. In this calculation we take into account the renormalization of the densities of states of the electrons and holes in bismuth due to scattering of these charge carriers on impurities. Without this renormalization, the amplitude of the oscillations of $S_{x y}^{(e)}(H)$ at low temperatures would be too large as compared with the amplitude observed in the experiment. $\underline{\underline{6}}$ As to the phonon-drag part $S_{x y}^{(p h)}(H)$ of the Nernst signal, this part has been taken into account within the simplest approximation. The results of the calculations of $S_{x y}=S_{x y}^{(e)}+S_{x y}^{(p h)}$ are compared with the experimental data, $\stackrel{6,10}{=}$ and we find a reasonable agreement between the theoretical and experimental curves in the region of the usual peaks of the Nernst coefficient $(H \leq 120 \mathrm{kOe})$. We also explain the lesser importance of the electrons than the holes in the production of the peaks and point out that measurements of the Nernst signal at lower temperatures would be useful for a more accurate comparison of the theory and the experiment. The approach of this paper, in principle, can also be used in analyzing the Nernst-coefficient oscillations recently observed in graphite $\underline{\underline{21}}$ 


\section{ACKNOWLEDGMENTS}

We thank K. Behnia for useful discussions and for providing us with the experimental data. This work was supported by the France-Ukraine program of scientific collaboration "DNIPRO".
1 K. Behnia, M.-A. Méasson, and Y. Kopelevich, Phys. Rev. Lett.98, 166602 (2007).

${ }^{2}$ K. Behnia, L. Balicas, and Y. Kopelevich, Science 317, 1729 (2007).

${ }^{3}$ Lu Li, J. G. Checkelsky, Y. S. Hor, C. Uher, A. F. Hebard, R. J. Cava, N. P. Ong, Science 321, 547 (2008).

4 Yu. V. Sharlai and G. P. Mikitik, Phys. Rev. B79, 081102(R) (2009).

5 J. Alicea and L. Balents, Phys. Rev. B79, 241101 (2009)

${ }^{6}$ H. Yang, B. Fauqué, L. Malone, A.B. Antunes, Z. Zhu, C. Uher, and K. Behnia, Nature Commun. 1, 47 (2010).

7 V.S. Edel'man, Usp. Fiz. Nauk 123, 257 (1977) [Sov. Phys. Usp. 20, 819 (1977)].

8 G.E. Smith, G.A. Baraff, and J.W. Rowell, Phys. Rev. 135, A1118 (1964).

${ }^{9}$ Here we use the value 0.07 for $M_{1}=M_{2}$ instead of 0.064 presented initially in Ref. 8 . This value better describes the oscillations of resistivity $\rho_{x x}(H)$ measured by S. G. Bompadre, C. Biagini, D. Maslov, and A. F. Hebard, Phys. Rev. B64, 073103 (2001).

${ }^{10}$ K. Behnia, M.-A. Measson, and Y. Kopelevich, Phys. Rev. Lett.98, 076603 (2007).
11 B.M. Askerov, Electron transport phenomena in Semiconductors (World Scientific, Singapore, 1994).

12 A.A. Abrikosov, Fundamentals of the Theory of Metals (North Holland, Amsterdam, 1988).

13 B. Fauque, H. Yang, I. Sheikin, L. Balicas, J.-P. Issi, and K. Behnia Phys. Rev. B79, 245124 (2009).

14 B. Fauque, B. Vignolle, C. Proust, J.-P. Issi, and K. Behnia, New J. Phys. 11113012 (2009).

15 Yu.N. Obraztsov, Fiz. Tverd. Tela (Leningrad) 7, 573 (1965) [Sov. Phys. Solid State 7, 455 (1965)].

16 D. L. Bergman and V. Oganesyan, Phys. Rev. Lett. 104, 066601 (2010).

17 P. S. Zyryanov and G. I. Guseva, Usp. Fiz. Nauk 95, 565 (1968) [Sov. Phys.-Uspekhi 11, 538 (1969)].

18 J.P. Jay-Gerin, Phys. Rev. B12, 1418 (1975).

19 Yu. A. Bychkov, Zh. Eksp. Teor. Fiz. 39, 1401 (1960)[Sov. Phys. JETP 12, 977 (1961)].

20 R. Hartman, Phys. Rev. 181, 1070 (1969).

21 Z. Zhu, H. Yang, B. Fauque, Y. Kopelevich, and K. Behnia, Nature Physics 6, 26 (2010). 